\title{
Treatment of lower extremity superficial vein thrombosis with low molecular weight heparin
}

\author{
Dzupina $\mathrm{A}^{1}$, Wald $\mathrm{M}^{2}$, Lee $\mathrm{BB}^{3}$, Stvrtinova $\mathrm{V}^{1}$ \\ 2nd Clinic of Internal Medicine, Faculty of Medicine Comenius University Bratislava, Slovakia. \\ alian@stonline.sk
}

\begin{abstract}
AIM: The aim of our study was to ascertain the efficacy of low-molecular-weight heparin (LMWH) in the treatment of patients with SVT.

MATERIAL AND METHODS: A group of 336 outpatients with clinical diagnosis of SVT was evaluated in this prospective study. At the beginning of the study all patients were examined by clinical investigation, laboratory tests and duplex ultrasound investigation (examination). All patients included into the study were treated with LMWH. Clinical and ultrasound evaluation was carried out on days 10, 30 and 90 of the follow-up. This clinical study of SVT treatment with LMWH was organized in 18 outpatient departments in the Slovak Republic. RESULTS: After 10 days of treatment with LMWH in full therapeutic dosage, an improvement in the clinical symptoms was demonstrated in $93 \%$ of patients; a complete resolution of clinical symptoms was demonstrated in $4 \%$ of patients. On day 30 (after 10 days of LMWH treatment in full therapeutic dosage and further treatment in halved therapeutic dosage up to 20 days) a complete resolution of clinical symptoms was observed in $59 \%$ $(n=189)$ of patients. Patients were further clinically evaluated on day 90 , after two months of no anticoagulation treatment. The clinical evaluation revealed a complete resolution of symptoms in $88 \%(n=283)$ of patients and improvement in symptoms in $11.6 \%(n=34)$. Two patients developed pulmonary embolization; extension of SVT was seen in one patient and SVT recurrence in two patients.

CONCLUSION: Superficial vein thrombosis can propagate into the deep veins with the risk of pulmonary embolism. The results indicate that current ambulatory treatment regimen using LMWH in the treatment of SVT is effective and safe (Fig. 4, Ref. 30). Text in PDF www.elis.sk.

KEY WORDS: lower extremity superficial vein thrombosis, low-molecular-weight heparin treatment.
\end{abstract}

\section{Introduction}

Superficial vein thrombosis (SVT) is a common and wellrecognized clinical entity characterized by a painful, warm, erythematous, tender, palpable cord-like structure along the course of a superficial vein, usually involving the lower extremities but potentially affecting any superficial vein in the body. SVT is an inflammation of the venous wall with subsequent or secondary clot formation. Therefore, the term superficial thrombophlebitis is used in literature, too. Nevertheless, the term superficial thrombophlebitis should be discouraged because inflammation and infection are not the only primary pathology. It should be called superficial vein thrombosis in order to avoid the unnecessary administration of antibiotics (1). It is not easy to distinguish the degree of inflammation and thrombosis, especially at the beginning of the disease. SVT occurs in two different forms, i.e. with varicose veins (varicophlebitis) and without varicose veins (non-varicose SVT). Non-varicose SVT is a group of miscellaneous disorders,

${ }^{12}$ nd Clinic of Internal Medicine, Faculty of Medicine Comenius University Bratislava, Slovakia, ${ }^{2}$ Department of Surgery, 2nd Faculty of Medicine, Charles University, Prague, Czech Republic, and ${ }^{3}$ Center for the Lymphedema and Vascular Malformations, George Washington University, Washington DC, USA while inflammation is a dominating feature in some conditions (superficial phlebitis), and thrombosis dominates in other cases (superficial thrombosis).

In many cases, SVT is a banal condition, which resolves spontaneously, thus the patient does not seek doctor's help. In literature, its incidence in the United States in 1973 has been estimated by clinical examination and extrapolation, to be 1 case of SVT per 1,950 people, and twenty years later in France in 1993 by duplex ultrasound investigation, to be one SVT per 220 people (2). These two studies differ a lot - not only in the incidence of SVT but especially in the method used for SVT diagnosis. The clinical diagnosis of SVT is easy as symptoms and signs are overt. But for precise evaluation of SVT, an extension compression duplex ultrasound (DUS) investigation is needed (3).

Some physicians consider SVT an integral part of venous thromboembolism, together with deep venous thrombosis (DVT) and pulmonary embolism (PE). PE in the elderly is an immediate threat of life (4). Due to a marked inflammatory reaction of the venous wall in SVT, the thrombus firmly adheres to the venous wall, thus the risk for pulmonary embolism is substantially smaller than in deep venous thrombosis. In the meta-analysis of twenty-one studies (4,358 patients) deep venous thrombosis was found in 18.1 $\%$ of SVT patients and in meta-analysis of 11 studies (2,484 patients) pulmonary embolism was found in $6.9 \%$ of SVT patients (5). 


\section{5-600}

The extent of thrombus seen during ultrasound investigation in the superficial vein is often much greater than clinically presumed. The extent of SVT seen on ultrasound is not only larger in the superficial vein, but the thrombus often continues extending into the deep venous system through perforating veins and/ or via sapheno-femoral or sapheno-popliteal junctions. Therefore, ultrasound investigation is essential for proper evaluation of the disease. In order to evaluate the deep venous system, other investigation methods, e.g imaging of the deep venous thrombosis using radioactive-labelled tirofiban, could be used in the future too (6).

The relationship between SVT and DVT is supported also by the same risk factors which trigger ST as well as DVT. Predisposing risk factors for SVT and VTE are similar and include varicose veins, immobilization, trauma, postoperative conditions, pregnancy, puerperium, active malignancies, auto-immune diseases, inherited thrombophilia, use of oral contraceptives or hormonal replacement therapy, advanced age, obesity, and history of previous VTE $(7,8)$.

Contrary to the treatment of DVT, only little is known about the most appropriate management of SVT. The aim of our study was to ascertain the efficacy of low-molecular-weight heparin (LMWH) treatment in patients with SVT diagnosed by duplex ultrasound investigation.

\section{Material and methods}

A total of 336 patients with the clinical diagnosis of SVT were included into this prospective study. The study was carried out in 18 out-patient departments of angiology, vascular surgery, internal medicine and haematology in Slovak Republic during the period of 17 months (between September 2009 and March 2011). The clinical study was approved by independent Ethics Committee/ Internal Review Board. Every patient signed informed consent for the study, including the recording of personal data.

At the beginning of the study, all patients were examined by clinical investigation, laboratory tests (blood count, basic biochemical analysis - AST, ALT, creatinine and other biochemical parameters based on clinical judgement of the doctor) as well as by duplex ultrasound (to confirm the length and localization of the thrombus in superficial veins and to exclude the thrombus in the deep and perforating veins).

Patients included into the study were all treated with low-molecular-weight heparin (LMWH) in a full anti-coagulant dose adjusted to patients' weight for 10 days, and then in halved anticoagulant dose for up to 30 days. The treatment included also compression treatment, topical treatment, venoactive drugs in case of varicophlebitis, and according to the decision of the specialist, also analgetics and antibiotics.

Clinical evaluations were carried out on days 10, 30 and 90 of the follow-up. Patients were assessed by DUS as needed or upon consideration of a specialist in charge. Haematology or biochemistry tests were performed based on the patient's clinical condition and physician's decision. The total length of follow-up was 90 days.

\section{Results}

Among the total of 336 patients enrolled in the study, 322 patients had met all evaluation criteria. Majority of the patients were women (72 \%), men accounted for $28 \%(n=94)$. The mean age was 58 years (youngest 32 years, oldest 76 years). $76 \%$ of patients were referred to the specialist by a general practitioner and the mean duration of symptoms from the onset until the visit to a specialist was 2 days (between 0 and 12 days). During DUS investigation of the patients, in 8 of them, concomitant DVT was revealed. DUS was not clearly positive in 5 patients who had thus not been included into the evaluation. Altogether 322 patients were included in the LMWH treatment and final evaluation.

The vast majority of patients (88\%) included in the study had SVT on a varicose vein (varicophlebitis), 51 patients had a history of DVT (16\%) and 119 patients (37\%) had a history of SVT.
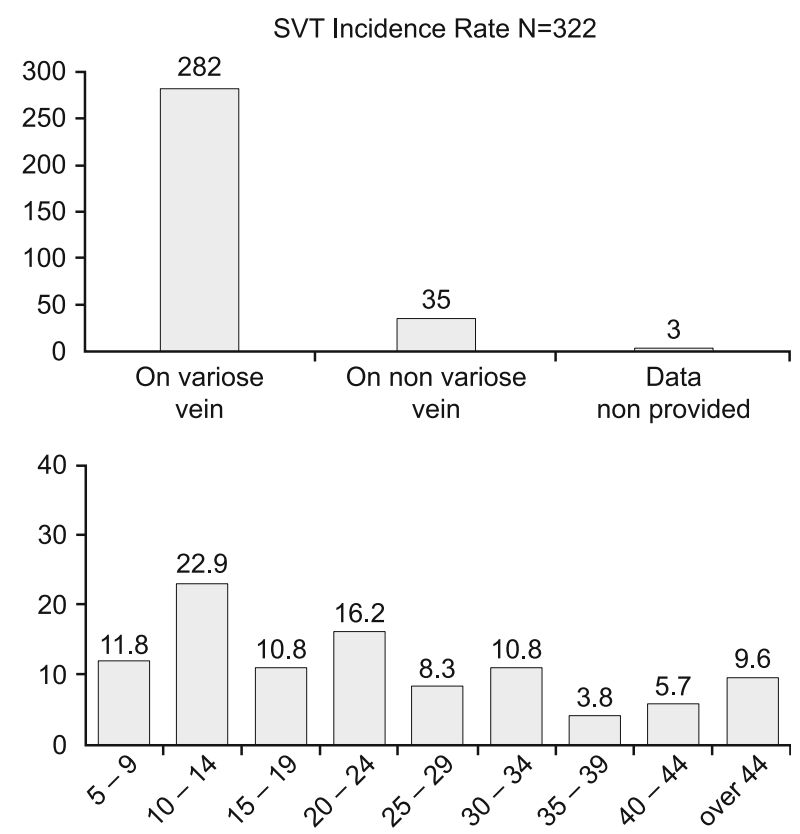

Fig. 1. Length of the thrombus in the superficial vein measured by DUS.

Disease status 30 days after treatment initiation $(\mathrm{N}=322)$

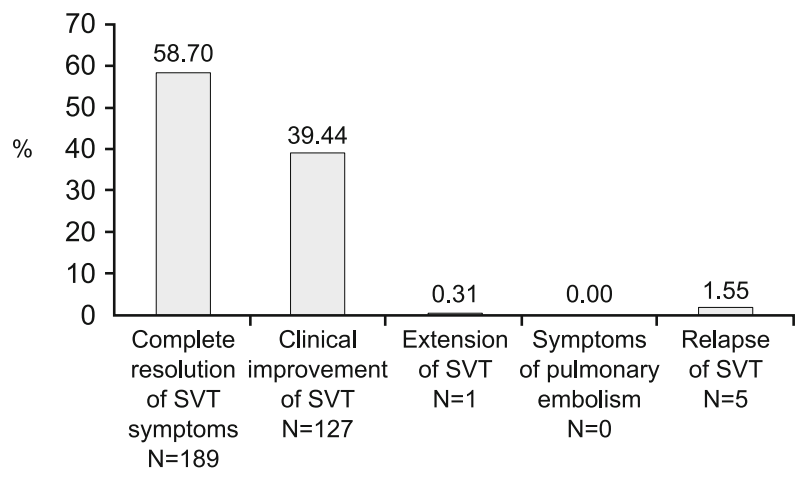

Fig 2. Clinical status after $\mathbf{3 0}$ days of anticoagulation treatment. 
Disease status 30 days after treatment initiation $(\mathrm{N}=322)$

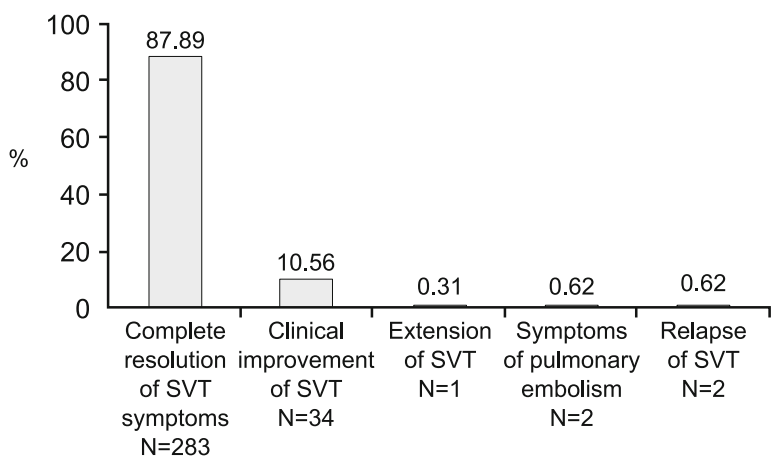

Fig. 3. Clinical status after 90 days of anticoagulation treatment.

Treatment continuation $(\mathrm{N}=322)$

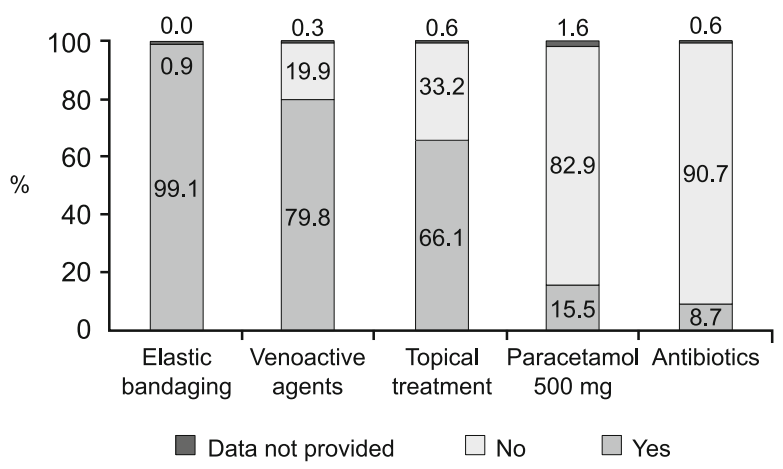

Fig 4. Treatment of SVT (apart from LMWH).

The average length of the thrombus in the superficial vein was $23 \mathrm{~cm}$ (with the minimum of $5 \mathrm{~cm}$ and maximum of $73 \mathrm{~cm}$ ) (Fig. 1). A complete occlusion of the vein lumen was observed in $73 \%$ of patients.

After 10 days of treatment with LMWH in full therapeutic dosage, the improvement in clinical symptoms was demonstrated in $93 \%$ of patients while complete resolution of clinical symptoms was demonstrated in $4 \%$ of patients. On day 30 (after 10 days of LMWH treatment in full therapeutic dosage and further treatment in halved therapeutic dosage up to 20 days) a complete resolution of clinical symptoms was observed in $59 \%(n=189)$ of patients (Fig. 2). Clinical improvement was reported in $40 \%$ $(n=127)$ of patients. Complications were reported in 6 patients, of which there was 1 extension of SVT (by $5 \mathrm{~cm}$ ), and 5 cases of disease recurrence (the same lower limb in 2 patients and the other leg in 3 patients). On day 30, the anticoagulation treatment with LMWH was ceased.

Patients were further clinically evaluated on day 90, after two months of no anticoagulation treatment. The clinical evaluation of patients revealed a complete resolution of symptoms in $88 \%$ (n $=283)$ of patients, improvement in symptoms in $10(6 \% ; n=34)$ (Fig. 3). Two patients developed pulmonary embolization; extension of SVT was seen in one patient and SVT recurrence in two patients. Serious impairment of liver and/or kidney function lead- ing to treatment discontinuation was not observed in any patient. No patient presented with heparin-induced thrombocytopenia, and the platelet count was within normal limits.

The treatment included also compression bandaging up to $99 \%$ ( $n=334$ ) of patients, venoactive drugs in $77 \%$ of patients (which is an expected high incidence regarding the high proportion of SVT on varicose veins), topical treatment in $67 \%$ of patients (Fastum gel or Liothon gel). Analgetics were used in $15 \%(n=50)$ and antibiotics in $8.3 \%(n=28)$ of patients (Fig. 4).

\section{Discussion}

There is no consensus on the optimal treatment of SVT in clinical practice. Several therapies have been proposed in literature, including surgical therapy (ligation or stripping of the affected veins), elastic stockings, non-steroidal anti-inflammatory drugs (NSAIDs) and different anticoagulants such as vitamin K antagonists, fondaparinux, LMWH and unfractionated heparin $(9,10,11,12)$. The aim of the treatment is to stop the extension in the superficial vein, to reduce the inflammation of the superficial vein and perivenous tissues as well as to prevent the extension of the thrombus formation into the deep and the communicating venous system. Unlike current clear definitions of treatment of deep-vein thrombosis, the regulations for treatment of superficial venous thrombosis are not yet clearly defined. At the level of evidence-based medicine, the data are relatively scarce and there are substantial discrepancies in recommendations from out-patient treatment through surgery, various treatment modalities with compression therapy and topical treatment, treatment with oral or parenteral non-steroidal anti-inflammatory agents (NSA) to heparins and LMWH in low, medium or high doses as well as various length of administration between 7 and 45 days.

In the recent study performed in the United Kingdom, a disparate management of SVT in primary and secondary care was found. The majority of clinicians (197 vascular surgeons and 172 general practitioners participated in this study) performed a venous duplex as their main investigation, but $40 \%$ performed no investigations at all. The majority of patients were treated with NSAIDs and only $25 \%$ with anticoagulation. Even fewer (less than $20 \%$ ) prescribed compression hosiery (13). This study demonstrates that the management of SVT is disparate despite good level of evidence indicating that NSADs, LMWH and compression hosiery are effective and reduce the incidence of DVT (9).

The main therapeutic procedure in all types of SVT is compression (elastic bandage or a compressive stocking) and mobilization. Everyday experience shows that compression of the thrombosed vein relieves the symptoms and speeds up healing. There have been no randomized studies demonstrating the effectiveness of compression, although this approach is considered essential by all experts. Fixative compression bandages used as the only treatment improved or eliminated the symptoms in $92.5 \%$ of patients while duplex ultrasound showed improvement in $81 \%$. No change or worsening on DUS was found in $19 \%$, and $3.8 \%$ of the patients developed DVT (14). Compression bandage is more appropriate 
than compression stocking mainly in the thigh location of SVT because it enables better compression of an affected vein, depending on individual local conditions in the thigh area (15). By pressure of superficial veins with elastic bandage, the further spread of inflammation to superficial veins is prevented, and acceleration of blood flow in deep veins is achieved. Thus the progression of inflammatory process into the deep vein system is prevented. Compression treatment reduces the pain and accelerates the healing process. A compressive stocking is beneficial in the prevention of recurrence of the disease. Compression treatment is essential for all patients with SVT (16). In our study, $99 \%$ of the patients were treated with compression (Fig. 4).

It is also absolutely inevitable to encourage ambulation (regular physical activity), rather than immobilization of the patient. Regular walking supports the effectiveness of the compression bandage on the lower extremity. The patient must walk regularly throughout the day and avoid prolonged periods of being seated or standing. Confinement to bed would favour the progression of the thrombus in both the superficial and the deep venous system and is therefore strictly contraindicated. Some authors judge the progression to the deep system as the prime complication (16).

Different studies included a LMWH as a treatment option in patients with SVT $(17,18,19)$. The rationale of treating SVT with LMWH is the neutralization and inhibition of thrombin generation and the prevention of PE and thrombus extension and thrombus recurrence. Anticoagulant therapy is recommended in patients with extensive SVT. LWMH, UFH (unfractionated heparin) or oral anticoagulants can be used in prophylactic or therapeutic doses. Treatment options regarding dose and duration differ in individual hospitals and medical care centres. A four-week LMWH treatment for SVT is associated with an incidence of HIT lower than $0.6 \%$ and platelet count monitoring may be omitted in this setting (20).

Both prophylactic and therapeutic LMWH given for 8 to 12 days were associated with a significantly lower incidence of SVT extension and/or recurrence, compared with placebo (OR 0.32; 95 $\%$ CI, 0.16 to 0.65 and OR 0.33; $95 \%$ CI, 0.16 to 0.58 , respectively) (21). Although the differences were not statistically significant, the incidence of venous thromboembolism (VTE) may have been lower both with prophylactic and therapeutic LMWH shortly after treatment (OR 0.25; and OR 0.26; $95 \%$ respectively) but not at the end of three-month follow-up period.

One study has compared two regimens of LMWH with each other (22). In a head-to-head comparison with one month therapeutic-dose, prophylactic-dose of LMWH, administered for the same period, led to a similar reduction in SVT extension and/ or recurrence and VTE events (OR 1.21; 95 \% CI, 0.39 to 3.78) over a three month follow up as therapeutic one. In the prophylactic LMWH group, most of VTE events (77\%) occurred while patients were still on treatment, whereas only $33 \%$ of patients on therapeutic-dose LMWH developed VTE during LMWH treatment. The advantage of therapeutic LMWH was lost after drug discontinuation.

The Belgian Society on Thrombosis and Haemostasis and the
Belgian Working Group of Angiology have good experience and good therapeutic results with 10 days of LMWH in full therapeutic dose followed by 20 days of LMWH in half-therapeutic dose (11). In our study we have used a similar therapeutic plan. Our patients included into the study were all treated with (LMWH) in full anti-coagulant dose adjusted to patients' weight for 10 days, and then in halved anti-coagulant dose for up to 30 days. One month after the beginning of treatment we have observed a complete resolution of clinical symptoms in $59 \%$ of patients and clinical improvement in $40 \%$ of patients (Fig. 2). Clinical evaluation three months after the beginning of study revealed a complete resolution of symptoms in $88 \%$ of patients and improvement in symptoms in 10; $6 \%$ (Fig. 3). Two patients developed pulmonary embolization, extension of SVT was seen in one patient and CVT recurrence in two patients.

One international randomized double-blind placebo-controlled study (CALISTO) assessed the use of fondaparinux, a synthetic selective inhibitor of factor Xa, in reducing the symptomatic VTE complications and/or death from any cause in patients with SVT of the lower limbs without concomitant DVT or $\mathrm{PE}$ at presentation. A significant $85 \%$ reduction in risk of subsequent VTE complications was observed in the fondaparinux group. These efficacy results were maintained 30 days after drug discontinuation (23). In Europe, fondaparinux is the only anticoagulant approved for the treatment of adults with acute symptomatic spontaneous SVT of the leg without concomitant DVT. But further analyses are needed to establish whether this therapeutic strategy is cost-effective (24).

According to the proceedings of the 9th ACCP (American College of Chest Physicians) Conference on Antithrombotic and Thrombolytic Therapy, SVT should be treated in modes as follows (25):

For patients with SVT of the lower limb of at least $5 \mathrm{~cm}$ in length, we suggest to use a prophylactic dose of fondaparinux or LMWH for 45 days over no anticoagulation (Grade 2B).

In patients with SVT who are treated with anticoagulation, we suggest fondaparinux, $2.5 \mathrm{mg}$ daily over a prophylactic dose of LMWH (Grade 2C).

Following recommendations concerning the treatment of SVT were published as a consensus statement of four international societies - Central European Vascular Forum (CEVF), Vasculab, International Union of Phlebology (IUP) and International Union of Angiology (IUA) (1):

All patients with SVT should be treated with compression therapy.

Immediate mobilization with elastic compression is mandatory. Patients should not be confined to bed.

Patients with SVT, with an inflamed and thrombosed superficial vein with thrombus longer than $5 \mathrm{~cm}$ on duplex ultrasound should have LMWH at intermediate or therapeutic dose for four weeks. The dosage and duration of anticoagulation depends on concomitant diseases and other risk factors for VTE.

In patients with extended SVT $(>10 \mathrm{~cm})$ with additional risk factors for VTE, fondaparinux in prophylactic dose could be considered for six weeks. 
Routine surgical ligation of SFJ or SPJ to prevent SVT extension into the deep veins is not advised.

As SVT is etiologically a heterogeneous group of disorders with a varying degree of inflammation and thrombosis, the main etiological factor and contribution of different risk factors should be considered before treatment decisions are taken (26). The use of antiplatelet agents is strongly recommended for the secondary prevention in elderly hospitalised bedridden polymorbid patients (27.) Venoactive agents are recommended in varicose SVT, although there is no clear data on the effects of this treatment. Antibiotics are indicated only in case of general signs of inflammation (fever, high white blood count, high sedimentation rate or C-reactive protein) or septic thrombophlebitis, for which the best treatment is a combination of aminoglykosides and broad-spectrum penicillins or cephalosporins. Corticosteroids are indicated only exceptionally in migrating thrombophlebitis, namely in cases of systemic diseases as thromboangiitis obliterans (28), morbus Behcet or morbus Mondor.

\section{Conclusion}

With the shift from clinical to ultrasonographic diagnosis and increased emphasis on the tenets of evidence-based medicine, SVT has attracted renewed clinical attention and interest in prognostic-therapeutic evaluation (29). SVT has long been considered to be a benign disease, which is to be managed with local and/ or systemic anti-inflammatory drugs in combination with elastic stockings. The diagnosis of SVT is primary clinical, but for precise evaluation of SVT extension, the compression duplex ultrasound investigation is needed. DUS allows evaluate not only the length of the thrombosis in the superficial vein but also the extension of the thrombus into the deep venous system.

A multicentre prospective clinical study of SVT treatment with LMWH was organized in 18 out-patient departments in the Slovak Republic during the period of 17 months. The results of our study indicate that current ambulatory treatment regimen using the combination of LMWH and compression treatment is effective and safe.

The main therapeutic procedure in all types of SVT is compression and mobilization. Non-steroidal anti-inflammatory drugs reduce painful symptoms and perivenous inflammation but there is no evidence that they reduce the incidence of thromboembolic events. Especially in cases of extensive SVT, anticoagulant therapy is a good choice. Several consensus groups have issued recommendations for more aggressive therapy, mainly with anticoagulants $(1,9,24,30)$. However, the relatively low grades of some of these recommendations may prompt questioning the real need to treat all patients with SVT with anticoagulants (19).

\section{References}

1. Kalodiki E, Stvrtinova V, Allegra C et al. Superficial vein thrombosis: a consensus statement. Int Angiol 2012; 31 (3): 203 - 216.

2. Ramelet AA, Perrin M, Kern P, Bounameaux H. Phlebology. Paris: Elsevier Masson SAS; 2008: 1-566.
3. Stvrtinova V, Poredos P, Allegra C. Superficial thrombophlebitis Consensus proposal from Central European Vascular Forum (CEVF) for diagnosis and treatment. Acta Phlebol 2011; 12 (5): 165-174.

4. Weberova D, Weber P. Meluzinova H, Matejovska-Kubesova $\mathbf{H}$, Polcarova V, Bielakova P, Canov P. Deceased elderly in-patients with pulmonary embolism. Bratisl Med J 2014; 115 (12): 786-790.

5. Di Minno MND, Ambrosino P, Ambrosisni E, Tremoli G, Di Minno G, Dentali F. Prevalence of deep vein thrombosis and pulmonary embolism in patients with superficial vein thrombosis: a systemic review and metaanalysis. J Thromb Haemost 2016; DOI:10.1111/jth.13279.

6. Darkovska Serafimovska M, Janevik-Ivanovska E, Ugresic M, Djorgoski I. Imaging of deep venous thrombosis using radioactive-labeled tirofiban. Bratisl Med J 2015; 116 (10): 621-626.

7. Barrelier MT. Superficial venous thrombosis of the legs. Phlebologie 1993; 46: 633-639.

8. De Moerloose P, Wutschert R, Heinzmann M, Perneger T, Reber G, Bounameaux H. Superficial vein thrombosis of lower limbs: influence of factor V Leiden, Factor II G20210 A and overweight. Thromb Haemost 1998; 80: 239-241.

9. Di Nisio M, Wichers IM, Middeldorp S. Treatment for superficial thrombophlebitis of the leg. Cochrane Database Syst Rev 2013; 4: CD004982.

10. Hirsch J. Guidelines for antithrombotic therapy. Hamilton: BC Decker Inc, 2008: 1- 180.

11. De Maeseneer MG. Superficial thrombophlebitis of the lower limb: practical recommendations for diagnosis and treatment. Acta Chir Belg 2005; 105 (2): 145-147.

12. Cosmi M. Management of superficial vein thrombosis. J Thromb Haemost 2015; 13: 1175-1183.

13. Lane TRA, Sritharan K, Herbert JR, Franklin IJ, Davies AH. The disparate management of superficial venous thrombosis in primary and secondary care. Phlebology 2015; 30: 172-179.

14. Mayer W, Partsch H. Superficial thrombophlebitis: A harmless disorder? Scope Phlebol Lymphol 1999; 2: 36-38.

15. Blättler W, Schwarzenbach B, Largiader J. Superficial vein thrombophlebitis - serious concern or much ado about little? VASA, 2008; 37: 31-38.

16. Partsch H, Rabe E, Stemmer R. Compression therapy of the extremities. Paris: Editions Phlebologiques Francaises; 2000: 1-398.

17. Kalodiki E, Nicolaides AN. Superficial thrombophlebitis and lowmolecular-weight heparins. Angiology 2002; 53 (6): 659-663.

18. Raffetto JD, Eberhardt RT. Benefit of anticoagulation for the treatment of lower extremity superficial venous thrombosis. J Vasc Surg: Venous Lym Dis 2015; 3: 236-241.

19. Décousus H, Bertoletti L, Frappé P. Spontaneous acute superficial vein thrombosis of the legs: do we really need to treat? J Thromb Haemost 2015; 13 (Suppl 1): S230-S237.

20. Sartori M, Favaretto E, Migliaccio L, Guazzaloca G, Legnani C, Palareti G, Cosmi B. The incidence of heparin - induced thrombocytopenia in patients treated with low molecular weight heparin for superficial vein thrombosis. Throm Res 2016; 139: 154-157.

21. The STENOX Study Group. A randomized double-blind comparison of low-molecular-weight heparin, a non-steroidal antiinflammatory agent and placebo in the treatment of superficial vein thrombosis. Arch Intern Med 2003; 163: 1657-1663. 


\section{$595-600$}

22. The VESALIO Investigators Group. High vs. low doses of lowmolecular-weight heparin for the treatment of superficial vein thrombosis of the legs: a double-blind, randomized trial. J Thromb Haemost 2005; 3: 1152-1157.

23. Decousus H, Prandoni P, Mismetti P et al. Fondaparinux for the treatment of superficial-vein thrombosis in the legs. N Engl J Med 2010; 363: $1222-1232$.

24. Blondon M, Righini M, Bounameaux H, Veenstra DL. Fondaparinux for isolated superficial vein thrombosis of the legs: a cost-effectiveness analysis. Chest 2012; 141: 321-329.

25. Kearon C, Akl EA, Comerota AJ et al. Antithrombotic Therapy for VTE Disease: Antithrombotic Therapy and Prevention of Thrombosis: American College of Chest Physicians Evidence-Based Clinical Practice Guidelines. Chest 2012; 141 (Suppl 2): e419S-494S.
26. Štvrtinová V, Dukát A, Džupina A et al. Superficial thrombophlebitis - diagnostics and treatment, Vaskulárna medicína, 2011; 3 (3): 123-129.

27. Wawruch M, Slezakova V, Murin $\mathbf{J}$ et al. The use of antiplatelet medication in hospitalised elderly patients. Bratisl Med J 2015; 116 (9): 533-538.

28. Štvrtinová V, Ambrózy E, Štvrtina S, Lesný P. 90 years of Burger`s disease - what was changed ? Bratisl Med J 1999; 100 (3): 123-128.

29. Allegra C. Superficial vein thrombosis. Acta Phlebol 2015; 16: 97-98.

30. Nicolaides A, Hull RD, Fareed J. Cardiovascular Disease Educational and Research Trust, European Venous Forum, North American Thrombosis Forum, International Union of Angiology and Union Internationale de Phlebologie, Superficial vein thrombosis. Clin Appl Thromb Hemost 2013; 19: 208-213. 